\title{
A retrospective analysis on the correlation between hip pain, physical examination findings, and alpha angle on MR images
}

\author{
Olcay Guler ${ }^{1 *}$, Mehmet Isyar ${ }^{1}$, Dilek Karataş ${ }^{2}$, Tugrul Ormeci ${ }^{3}$, Halis Cerci ${ }^{4}$ and Mahir Mahirogulları ${ }^{1}$
}

\begin{abstract}
Background: We aimed to search whether alpha angle, a radiological clue used in the diagnosis of femoroacetabular impingement, is correlated with the presence of hip pain, internal rotation angle, and impingement test results on hip impingement patients (CAM type).

Methods: Medical records of 334 patients (156 women, 178 men) with an average age of $33.8 \pm 8.4$ (range 20-50) years were retrospectively studied for the alpha angle of the hip measured on magnetic resonance images (MRI). Hip pain and internal rotation angles as well as results of impingement tests were reviewed.

Results: Hip pain was reported more frequently on the right side $(n=35,10.5 \%)$ compared to the left side $(n=$ $22,6.6 \%)(p=0.047)$. No difference was observed between the right and left sides regarding alpha angles ( $p=0$. $145)$, internal rotation angles ( $p=0.637)$, or positivity of impingement test $(p=0.210)$. Internal rotation angles were significantly higher in cases without hip pain $(p<0.001)$ and in patients with negative impingement test result $(p<0.001)$. Internal rotation angle correlated positively with age and negatively with the alpha angle. Alpha angle was increased in cases that report pain, those with an internal rotation angle $<20^{\circ}$, or cases with positive impingement test. The pain was more common, internal rotation angle was higher, and positivity for impingement was more frequent if the alpha angle was $<55^{\circ}$. Patients with hip pain or positive impingement test or internal rotation angle $<20^{\circ}$ had increased alpha angles $(p<0.001)$.

Conclusions: The pain, impingement test results, and internal rotation angle seem to be associated with alpha angle of the hip measured on MRI in hip impingement patients.
\end{abstract}

Keywords: Femoroacetabular impingement, Alpha angle, Internal rotation, Hip, Pain

\section{Background}

Femoroacetabular impingement (FAI) is a morphological disorder of the hip joint that shares a similar mechanical etiology with osteoarthritis [1]. A certain amount of idiopathic hip arthritis cases may be linked with FAI [1]. This pathology may occur due to bone abnormalities caused by overcoverage of the acetabulum (pincer type), asphericity of the femoral head and neck (cam type), or a combination of these conditions [2]. Femoroacetabular impingement may trigger cartilage destruction and give

\footnotetext{
*Correspondence: olcayguler77@gmail.com

${ }^{1}$ Orthopedics and Traumatology Department, Medical Faculty, Medipol University, Atatürk Bulvarı No: 27 Unkapanı, 34083 Fatih, Istanbul, Turkey Full list of author information is available at the end of the article
}

rise to osteoarthritis $(\mathrm{OA})$ of the hip, which may present clinically as hip pain and restriction of movement [3].

The alpha angle is defined by Nötzli et al. to evaluate the asphericity of the head of the femur in magnetic resonance image (MRI) views [4]. It allows the assessment of the contour deformity of the femoral headneck junction and may aid in setting the guidelines for treatment [5].

Timely diagnosis and appropriate treatment are crucial for the reduction of pain, improvement of function and prevention, or at least delay of OA. Early detection is especially important since the restoration of function may not be feasible after end-stage OA has occurred. Although early diagnosis is particularly important, misdiagnosis by clinicians unfamiliar with the disease is not 
uncommon. Therefore, integrated assessment of clinical and radiological findings is imperative for the identification of FAI. From this viewpoint, the definition of practical, reliable, and useful radiological tips and elucidation of the relationship between descriptive, clinical, and radiological variables will be critical to avoid a delay in diagnosis $[1,6]$.

In addition to radiological measures, clinical examination is crucial for screening asymptomatic abnormalities of FAI. The impingement test is highly sensitive to the induction of hip pain in symptomatic FAI patients. Symptomatic FAI patients frequently exhibit a limited range of motion involving flexion, abduction, adduction, and internal and external rotation [7]. Moreover, internal and external rotation and abduction are significantly correlated to alpha angle in symptomatic FAI patients [8]. However, whether these tests can be used on a population that is largely asymptomatic and only complains of hip pain for diagnostic and screening purposes is unclear.

The aim of the present study was to determine the factors affecting hip pain, internal rotation angle, alpha angle, and impingement test results and to investigate a correlation between alpha angle and these parameters in FAI patients (CAM type).

\section{Methods}

\section{Study design}

This retrospective study was implemented to study the relationship between impingement test, hip alpha angle, and hip pain for 3 months by using data derived from the medical files of 378 FAI patients (aged 20 to 50 years) admitted to the orthopedics and traumatology department of our tertiary care center and had hip MRI examination. Approval from the local Institutional Review Board was obtained before the study (Istanbul Medipol University Ethics Committee, date 25/06/2015, no 108400987-358).

Exclusion criteria were as follows: the presence of rheumatologic diseases (2 patients), arthrosis causing narrowing of the joint (3 patients), the presence of chondral injury or labrum tear on magnetic resonance images (MRI) (11 patients), pregnancy, history of previous hip surgery ( 5 patients) or hip disease during childhood (1 patient), lateral central edge angle was $<20^{\circ}$ on anteroposterior hip $\mathrm{x}$-ray examination (6 patient), crossover sign, coxa profunda or protrusio acetabuli on anteroposterior pelvic $\mathrm{x}$-ray examination (9 patient), retrovert acetabuli (4 patient), and femoral internal rotation angle $>60^{\circ}$ while on physical examination in supine position (3 patient). As a result, 44 patients were excluded and 334 patients were included to the study.

\section{Impingement test}

The impingement test results were derived from the medical files of the patients. The test was performed in supine position by the same orthopedician. The hip joint was brought to passive flexion at $90^{\circ}$, together with adduction and internal rotation. A verbal report of pain during this maneuver was interpreted as a positive test result [9].

\section{Internal rotation angle}

The degree of internal rotation was derived from the medical files of the patients. Physical examination was performed in supine position by the same orthopedician. The degree of internal rotation was measured using a goniometer while the hip was at $90^{\circ}$ flexion. Abduction and adduction of the hip were limited due to pressure applied downward on the knee. The ankle was used to rotate the hip internally and externally [10].

\section{Magnetic resonance imaging}

All imaging studies were conducted on a 1.5-Tesla MRI device (Sigma HDXT, General Electric, Chicago, IL, USA). Patients were in supine position while the hip joint was maintained in neutral position. Pulse sequence parameters of the turbo spin-echo sequence were as follows: repetition time (Tr), $637 \mathrm{~ms}$; echo time (Te), $14 \mathrm{~ms}$; field of view (FOV), $350 \times 350 \mathrm{~mm}$; matrix, $512 \times$ 256; slice thickness, $3 \mathrm{~mm}$; flip angle, $150^{\circ}$. In addition, we used a coronal T1-weighted sequence $(\mathrm{Tr}, 530 \mathrm{~ms}$; Te, $14 \mathrm{~ms}$; FOV, $400 \times 400 \mathrm{~mm}$; slice thickness, $5 \mathrm{~mm}$; flip angle, $150^{\circ}$ ), axial oblique T1-weighted sequence (Tr, $530 \mathrm{~ms}$; Te, $14 \mathrm{~ms}$; FOV, $350 \times 265 \mathrm{~mm}$; slice thickness, $5 \mathrm{~mm}$; flip angle, $150^{\circ}$ ) oriented along the axis of the femoral neck, and fat-suppressed T1-weighted fast low angle shot (FLASH) sequences ( $\mathrm{Tr}, 795 \mathrm{~ms}$; Te, $11 \mathrm{~ms}$; FOV, $400 \times 400 \mathrm{~mm}$; slice thickness, $3 \mathrm{~mm}$; flip angle, $60^{\circ}$ ).

\section{Measurement of alpha angles on MRI views}

Alpha angle was measured separately by two radiologists (DK, TO) who were blinded to patient data using the method described by Nötzli et al. [4] For this purpose, the Picture Archiving and Communication System (PACS, General Electric, Chicago, IL, USA) was used. Accordingly, the alpha angle of the hip was defined as the angle between two intersecting lines at the center of the femoral head. Using a best-fit circle digitized around the femoral head, the first line was extended from the center of the femoral head to the mid-point of the femoral neck. The second line was drawn from the center of the femoral head to the deviation of the femoral neck from the circle drawn around the femoral head (Fig. 1).

\section{Statistical analysis}

A total of 334 cases (668 hips) were recruited. As the expected prevalence was reported to be $15 \%$, this number allowed us to estimate the $95 \%$ confidence interval (CI) 


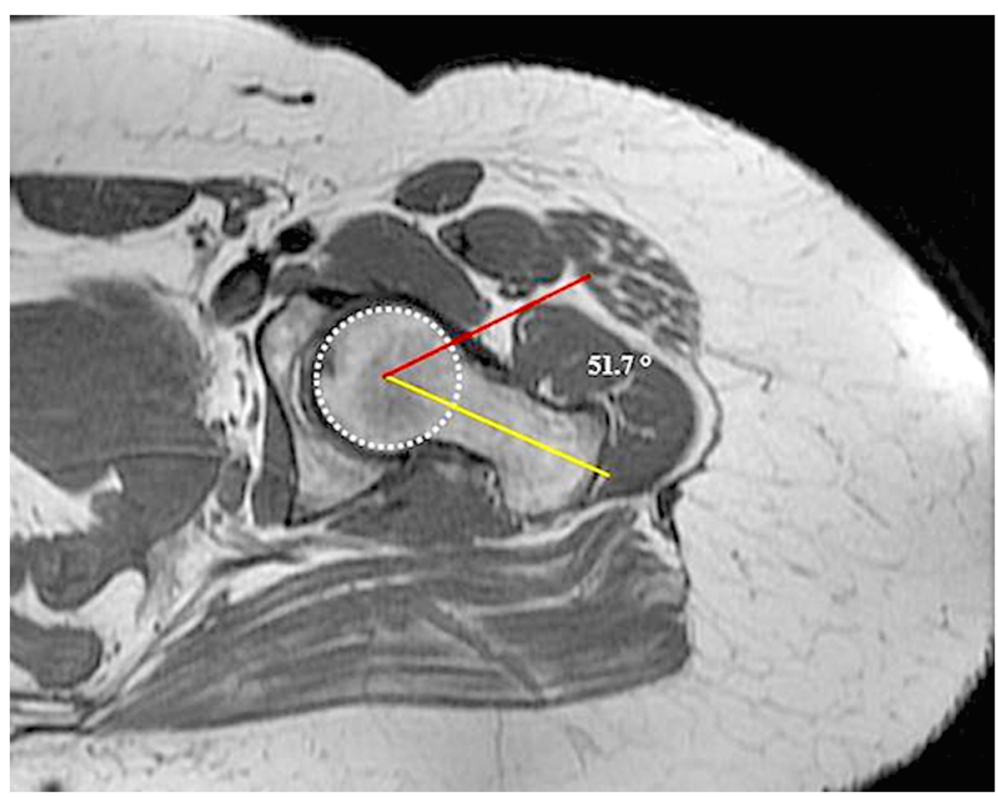

Fig. 1 Measurement of alpha angle on MRI view of the left hip of a 24-year-old woman (the yellow line extends from the center of the femoral head to the midpoint of the femoral neck; the red line is extended from the center of the femoral head to the deviation of the femoral neck from the circle drawn around the femoral head)

with a $5 \%$ margin of error and power of $90 \%$ [11]. Descriptive statistics (mean, standard deviation, median, minimum, and maximum) were used for continuous variables. Qualitative variables were compared with the chisquare test, while categorical dependent variables were studied using the McNemar test. Two independent groups that were not distributed normally were compared using the Mann-Whitney $U$ test. Student's $t$ test was used to evaluate two independent groups with a normal distribution. The Wilcoxon Signed Rank test was used to compare two dependent variables that were not distributed normally. The level of significance was set at $p<0.05$, and all analyses were implemented using the MedCalc Statistical Software program version 12.7.7 (MedCalc Software bvba, Ostend, Belgium; http://www.medcalc.org; 2013). Cronbach's alpha, which indicates the average inter-correlation for the two radiologists who carried out measurements for the right and left hips, were 0.932 and 0.928 , respectively.

\section{Results}

An overview of the data is shown in Tables 1, 2, and 3 . Our series consisted of 156 women (46.7\%) and 178 men (53.3\%), with an average age of $33.8 \pm 8.4$ years (range, 20 to 50 ). No difference was detected between women and men regarding age $(p=0.885)$. Average alpha angle was $53.1 \pm 1.9$ (range, 48.9 to 62.0 ). There was no significant difference between the right and left sides $(p=0.145)$.
Report of pain in the right hip (10.5\%) was more frequent than in the left hip (6.6\%) $(p=0.047)$, but results of the impingement test $(p=0.210)$ and internal rotation $(p=0.637)$ were similar. For both hips, the angle of internal rotation was significantly increased in the absence of hip pain and in patients with a negative impingement test ( $p<0.001$ for both).

In cases with an angle of internal rotation $<20^{\circ}$, alpha angle and the likelihood of positivity of the impingement test were significantly increased $(p<0.001$ for both). There was a moderate and negative correlation between internal rotation and alpha angle $(r=-0.555 ; p<0.001)$.

The pain was reported more frequently in patients with positive impingement test results $(p<0.001)$. Patients that reported pain were younger $(p=0.040)$ and had increased alpha angles $(p<0.001)$.

Cases with alpha angles $\geq 55^{\circ}$ were younger $(p=$ $0.005)$, suffered more frequently from pain $(p<0.001)$, and were more likely to have positive impingement test

Table 1 Alpha angle, hip pain, positive impingement test, and internal rotation angle in the right and left hips

\begin{tabular}{llll}
\hline Variable & \multicolumn{2}{l}{ Hip involvement } & $p$ value \\
\cline { 2 - 3 } & Right & Left & \\
\hline Alpha angle & $53.2 \pm 2.0$ & $53.1 \pm 2.0$ & 0.145 \\
Hip pain (yes/no) & $35 / 299$ & $32 / 302$ & 0.047 \\
Positive impingement test & $14 / 344$ & $8 / 334$ & 0.210 \\
Internal rotation angle & $31.7 \pm 10.0$ & $31.8 \pm 9.4$ & 0.637 \\
\hline
\end{tabular}


Table 2 Average values for internal rotation angles with respect to the presence of hip pain and positive impingement test

\begin{tabular}{llllllll}
\hline Side & \multicolumn{1}{l}{ Hip pain } & & $p$ value & \multicolumn{2}{l}{ Impingement test } & $p$ value \\
\cline { 2 - 3 } \cline { 6 - 7 } & $(-)$ & $(+)$ & & $(-)$ & $(+)$ & \\
\hline Right & $33.6 \pm 8.5$ & $15.3 \pm 5.3$ & $<0.001$ & $32.4 \pm 9.6$ & $16.8 \pm 5.8$ & $<0.001$ \\
Left & $32.9 \pm 8.6$ & $16.8 \pm 7.5$ & $<0.001$ & $32.2 \pm 9.1$ & $16.3 \pm 6.4$ & $<0.001$ \\
\hline
\end{tabular}

results $(p<0.001)$. The degree of internal rotation was higher in cases with alpha angles $<55^{\circ}(p<0.001)$.

\section{Discussion}

The purpose of the present study was to determine whether alpha angle was correlated with the presence of hip pain, internal rotation angle, and impingement test results in FAI patients (CAM type). Our results indicated that hip pain occurred more frequently on the right side, and internal rotation angles were significantly higher in cases that did not report hip pain and in those with a negative impingement test result. Internal rotation angle correlated positively with age and negatively with the alpha angle. Alpha angle was increased in cases that reported pain, those with an internal rotation angle $<20^{\circ}$, or cases with a positive impingement test. The pain was more common, internal rotation angle was higher, and there were a greater number of positive impingements when the alpha angle was $<55^{\circ}$. Patients with hip pain or a positive impingement test or an internal rotation angle $<20^{\circ}$ had significantly increased alpha angles. These results imply that alpha angle may be associated with clinical parameters and thus, may serve as a valuable marker for screening and diagnosing patients having complaints consistent with FAI.

Active and young people may suffer from groin pain due to internal rotation of the hip at $90^{\circ}$ flexion. In such circumstances, the likelihood of anterior impingement of the femoral neck on the acetabular rim or labrum must be taken into account [12].

Osteoarthritis of the hip is a multifactorial disease linked to systemic and local risk factors such as degeneration due to slipped capital femoral epiphysis, developmental dysplasia of the hip, and Legg-Calvé-Perthes disease [13]. Nevertheless, these theories cannot fully explain cases with early OA. Some subtle structural alterations in the proximal femur or acetabulum have been associated with OA of the hip [11]. Since only a few data exist regarding the prevalence of radiographic changes in asymptomatic individuals, elucidation of the natural course of and its link with OA have not been possible to date [11].

Alpha angle was initially described by Notzli as an indicator of the loss of femoral head sphericity and, therefore, a marker of cam-type FAI [4]. A cut-off value was randomly determined between normal and abnormal angles; values $>50^{\circ}$ were considered abnormal [4]. The lack of uniformity in this criterion is reflected in our results, and similar to a report by Diesel, the cutoff value for alpha angle in our series was determined to be $55^{\circ}$ [12]. In the literature, it has been reported that the cut-off value for a normal alpha angle can range from $42^{\circ}$ to $68^{\circ}$ [14].

Analysis of the relationship between hip deformities and the risk of OA development revealed that the nonspherical shape of the femoral head and enlargement of the femoral neck were linked with increased risk. However, these alterations may be a consequence of OA rather than a cause [15].

Our results were in accordance with the report by Kapron et al., suggesting that internal rotation measured in the supine position negatively correlates with alpha angle [2]. Pain may be elicited only in cases with underlying chondrolabral damage, and therefore, it must be remembered that the impingement test may be used to distinguish underlying chondrolabral damage [13].

Barton et al. suggested that radiographs might be sufficient for evaluation of the femoral head-neck junction [5]. In FAI, diagnostic imaging should be considered as a complementary measure to the clinical evaluation rather than being the sole tool for diagnosis [16]. The cam deformity may be diffuse along the femoral head-neck junction rather than being focal [17].

Magnetic resonance imaging is a specific imaging tool used to assess groin and hip pain. Since there is no standard test for such abnormalities, we hope that alpha angle may aid in the determination of the femoral headneck relationship on MRI scans. Although there is much debate over the radiographic cut-off value for FAI diagnosis, we hope that our efforts will aid in establishing a standard screening protocol. From this point of view, the cut-off value of $55^{\circ}$ for alpha angle and the negative correlation between internal rotation and alpha angle are important. Moreover, hip pain, impingement test

Table 3 Alpha angle, presence of hip pain, and positive impingement test in various subgroups

\begin{tabular}{|c|c|c|c|c|c|c|c|c|c|}
\hline & \multicolumn{2}{|c|}{ Internal rotation angle } & \multirow[t]{2}{*}{$p$ value } & \multicolumn{2}{|c|}{ Impingement test } & \multirow[t]{2}{*}{$p$ value } & \multicolumn{2}{|l|}{ Hip pain } & \multirow[t]{2}{*}{$p$ value } \\
\hline & $\overline{<20^{\circ}}$ & $\geq 20^{\circ}$ & & $(-)$ & $(+)$ & & $(-)$ & $(+)$ & \\
\hline Alpha angle & $56.7 \pm 2.3$ & $52.8 \pm 1.6$ & $<0.001$ & $53.0 \pm 1.8$ & $56.1 \pm 2.6$ & $<0.001$ & $52.7 \pm 1.5$ & $55.9 \pm 2.2$ & $<0.001$ \\
\hline Hip pain (yes/no) & $22 / 26$ & $25 / 283$ & $<0.001$ & $31 / 315$ & $16 / 19$ & $<0.001$ & - & - & \\
\hline Positive impingement test & $10 / 6$ & 9/299 & $<0.001$ & - & - & & - & - & \\
\hline
\end{tabular}


positivity, and an internal rotation angle $<20^{\circ}$ were associated with increased alpha angle. A cautious and integrative assessment of physical examination findings and radiological data is mandatory to establish timely and correct diagnoses. Our findings imply that alpha angle is correlated with hip pain, and impingement test results and internal rotation and assessment of alpha angle in patients complaining of hip or groin pain may yield valuable data for both treatment and follow-up. Even though the accuracy of the alpha angle measurement on plain radiographs has been shown, MRI still stays as a powerful diagnostic measure that provides prognostic information and allows patient counseling [5].

Limitations of the present study include the lack of radiographic cut-off values and possible influences of genetic, ethnic, and environmental factors on the parameters under investigation. Reliability of the measurements with a goniometer is doubtful due to the personal variability of the anatomic landmarks. Moreover, measurements of internal rotation were not performed in prone and sitting positions. It must be noted that this study reflects the experience of a single institution, and the possibility of error and bias cannot be completely eliminated.

\section{Conclusions}

The pain, impingement test results, and internal rotation angle seem to be associated with alpha angle of the hip measured on MRI in hip impingement patients.

\section{Abbreviatıons}

FAl: Femoroacetabular impingement; MRI: Magnetic resonance images; OA: Osteoarthritis

\section{Acknowledgements}

The English in this document has been checked by at least two professional editors, both native speakers of English.

\section{Funding}

No financial support was received for this paper.

\section{Availability of data and materials}

All analyses were implemented using the MedCalc Statistical Software program version 12.7.7 (MedCalc Software bvba, Ostend, Belgium; http://www.medcalc.org; 2013).

\section{Authors' contributions}

OG carried out the data collection and study design. MI carried out the statistical analysis. DK and TO carried out the radiological evaluation and measurement. $\mathrm{HC}$ carried out and participated in the manuscript design. MM carried out the edition and study design. All authors read and approved the final manuscript.

\section{Competing interests}

The authors declare that they have no competing interests.

\section{Ethics approval and consent to participate}

This study was approved by the Institutional Ethics Committee (Istanbul Medipol University Ethics Committee, date 25/06/2015, no 108400987-358) and conducted in accordance with the latest version of the Helsinki Declaration. All patients were informed of the study and signed a written informed consent form.

\section{Author details}

'Orthopedics and Traumatology Department, Medical Faculty, Medipol University, Atatürk Bulvarı No: 27 Unkapanı, 34083 Fatih, Istanbul, Turkey. ${ }^{2}$ Department of Radiology, Nisa Hospital, Istanbul, Turkey. ${ }^{3}$ Department of Radiology, Medical Faculty, Medipol University, Istanbul, Turkey. ${ }^{4}$ Department of Orthopedics and Traumatology, Nisa Hospital, Istanbul, Turkey.

Received: 11 August 2016 Accepted: 7 November 2016

Published online: 15 November 2016

\section{References}

1. Lahner M, von Schulze Pellengahr C, Walter PA, et al. Biomechanical and functional indicators in male semiprofessional soccer players with increased hip alpha angles vs. amateur soccer players. BMC Musculoskelet Disord. 2014;15:88.

2. Kapron $\mathrm{AL}$, Anderson $\mathrm{AE}$, Peters $\mathrm{CL}$, et al. Hip internal rotation is correlated to radiographic findings of cam femoroacetabular impingement in collegiate football players. Arthroscopy. 2012;28:1661-70.

3. Tanzer M, Noiseux N. Osseous abnormalities and early osteoarthritis: the of hip impingement. Clin Orthop Relat Res. 2004:429:170-7.

4. Nötzli HP, Wyss TF, Stoecklin CH, et al. The contour of the femoral headneck junction as a predictor for the risk of anterior impingement. J Bone Joint Surg Br. 2002;84:556-60.

5. Barton C, Salineros MJ, Rakhra KS, et al. Validity of the alpha angle measurement on plain radiographs in the evaluation of cam-type femoroacetabular impingement. Clin Orthop Relat Res. 2011;469:464.

6. Larson CM, Giveans MR, Taylor M. Does arthroscopic FAl correction improve function with radiographic arthritis? Clin Orthop Relat Res. 2011;469:1667-76.

7. Philippon MJ, Maxwell RB, Johnston $\mathrm{TL}$, et al. Clinical presentation of femoroacetabular impingement. Knee Surg Sports Traumatol Arthrosc. 2007; 15:1041-7.

8. Johnston TL, Schenker ML, Briggs KK, et al. Relationship between offset angle alpha and hip chondral injury in femoroacetabular impingement. Arthroscopy. 2008;24:669-75.

9. Gosvig KK, Jacobsen S, Sonne-Holm S, et al. Prevalence of malformations of the hip joint and their relationship to sex, groin pain, and risk of osteoarthritis: a population-based survey. J Bone Joint Surg Am. 2010;92: 1162-9.

10. Wyss TF, Clark JM, Weishaupt D, et al. Correlation between internal rotation and bony anatomy in the hip. Clin Orthop Relat Res. 2007;460:152-8.

11. Leunig $M$, Beck $M$, Dora $C$, et al. Femoroacetabular impingement: trigger for the development of coxarthrosis. Orthopade. 2006;35:77-84.

12. Economopoulos KJ, Milewski MD, Hanks JB, et al. Radiographic evidence of femoroacetabular impingement in athletes with athletic pubalgia. Sports Health. 2014;6:171-7.

13. Diesel CV, Ribeiro TA, Scheidt RB, et al. The prevalence of femoroacetabular impingement in radiographs of asymptomatic subjects: a cross-sectional study. Hip Int. 2015;25:258-63.

14. Pollard TC, Villar RN, Norton MR, et al. Femoroacetabular impingement and classification of the cam deformity: the reference interval in normal hips. Acta Orthop. 2010;81:134-41.

15. Doherty M, Courtney P, Doherty S, et al. Nonspherical femoral head shape (pistol grip deformity), neck shaft angle, and risk of hip osteoarthritis: a casecontrol study. Arthritis Rheum. 2008;58:3172-82.

16. Beaule PE, Allen DJ, Clohisy JC, et al. The young adult with hip impingement: deciding on the optimal intervention. J Bone Joint Surg Am. 2009;91:210-21.

17. Rakhra K, Sheikh AM, Allen DJ, et al. Comparison of MRI alpha angle measurement planes in femoroacetabular impingement. Clin Orthop Relat Res. 2009:467:660-5. 